\title{
Motion-corrected 3D whole-heart water-fat high-resolution late gadolinium enhancement cardiovascular magnetic resonance imaging
}

Camila Munoz $^{1 *}$ (D), Aurelien Bustin ${ }^{1}$, Radhouene Neji ${ }^{1,2}$, Karl P. Kunze $^{2}$, Christoph Forman $^{3}$, Michaela Schmidt ${ }^{3}$, Reza Hajhosseiny ${ }^{1}$, Pier-Giorgio Masci ${ }^{1}$, Martin Zeilinger ${ }^{4}$, Wolfgang Wuest ${ }^{4}$, René M. Botnar ${ }^{1}$ and Claudia Prieto ${ }^{1}$

\begin{abstract}
Background: Conventional 2D inversion recovery (IR) and phase sensitive inversion recovery (PSIR) late gadolinium enhancement (LGE) cardiovascular magnetic resonance (CMR) have been widely incorporated into routine CMR for the assessment of myocardial viability. However, reliable suppression of fat signal, and increased isotropic spatial resolution and volumetric coverage within a clinically feasible scan time remain a challenge. In order to address these challenges, this work proposes a highly efficient respiratory motion-corrected 3D whole-heart water/fat LGE imaging framework.
\end{abstract}

Methods: An accelerated IR-prepared 3D dual-echo acquisition and motion-corrected reconstruction framework for whole-heart water/fat LGE imaging was developed. The acquisition sequence includes 2D image navigators (iNAV), which are used to track the respiratory motion of the heart and enable $100 \%$ scan efficiency. Non-rigid motion information estimated from the 2D iNAVs and from the data itself is integrated into a high-dimensional patchbased undersampled reconstruction technique (HD-PROST), to produce high-resolution water/fat 3D LGE images. A cohort of 20 patients with known or suspected cardiovascular disease was scanned with the proposed 3D water/fat LGE approach. 3D water LGE images were compared to conventional breath-held 2D LGE images (2-chamber, 4chamber and stack of short-axis views) in terms of image quality (1: full diagnostic to 4: non-diagnostic) and presence of LGE findings.

Results: Image quality was considered diagnostic in 18/20 datasets for both 2D and 3D LGE magnitude images, with comparable image quality scores (2D: $2.05 \pm 0.72$, 3D: $1.88 \pm 0.90, p$-value $=0.62$ ) and overall agreement in LGE findings. Acquisition time for isotropic high-resolution $\left(1.3 \mathrm{~mm}^{3}\right.$ ) water/fat LGE images was $8.0 \pm 1.4 \mathrm{~min}$ (3-fold acceleration, 60-88 slices covering the whole heart), while 2D LGE images were acquired in $5.6 \pm 2.2 \mathrm{~min}(12-18$ slices, including pauses between breath-holds) albeit with a lower spatial resolution $(1.40-1.75 \mathrm{~mm}$ in-plane $\times 8 \mathrm{~mm}$ (Continued on next page)

\footnotetext{
* Correspondence: camila.munoz@kcl.ac.uk

'School of Biomedical Engineering and Imaging Sciences, King's College London, St Thomas' Hospital, 3rd Floor, Lambeth Wing, London SE1 7EH, UK Full list of author information is available at the end of the article
} 
(Continued from previous page)

slice thickness).

Conclusion: A novel framework for motion-corrected whole-heart 3D water/fat LGE imaging has been introduced. The method was validated in patients with known or suspected cardiovascular disease, showing good agreement with conventional breath-held 2D LGE imaging, but offering higher spatial resolution, improved volumetric coverage and good image quality from a free-breathing acquisition with 100\% scan efficiency and predictable scan time.

Keywords: 3D whole-heart, Water/fat cardiovascular magnetic resonance imaging, Late gadolinium enhancement, Motion-correction, Accelerated imaging

\section{Background}

Late gadolinium enhancement (LGE) cardiovascular magnetic resonance (CMR) imaging is widely accepted as the reference technique for the assessment of myocardial viability [1-3]. Inversion recovery (IR), and more recently phase-sensitive IR (PSIR) [4], 2D LGE imaging protocols have been introduced into routine CMR examinations for the detection and quantification of myocardial scar and fibrosis in both ischemic and non-ischemic cardiovascular disease.

In clinical routine, LGE images are conventionally acquired under repeated breath-holds as a series of 2D slices, with reasonably high in-plane spatial resolution (1.4 to $1.8 \mathrm{~mm}$ ) but large slice thickness of 6 to $8 \mathrm{~mm}$ and with $2-4 \mathrm{~mm}$ interslice gaps resulting in incomplete volumetric coverage [5, 6]. Recent technical developments have advanced the capabilities of LGE imaging for the depiction of small or patchy patterns of fibrosis, by focusing on improving spatial resolution and volumetric coverage. This is typically achieved by acquiring 3D datasets under free breathing, relying on diaphragmatic navigator based gating for respiratory motion compensation [7-14]. While this approach produces good-quality high-resolution images for subjects with regular breathing patterns, large variations in the respiratory efficiency among patients may lead to long and unpredictable scan times and decreased image quality, hindering its implementation in the clinical practice. Nevertheless, these approaches have been shown to produce images with smaller slice thickness (around 4 $\mathrm{mm}$ ), and more recently achieving near $2 \mathrm{~mm}$ isotropic resolution [12]. Furthermore, diaphragmatic navigated high-resolution 3D LGE imaging with $1.4 \mathrm{~mm}^{3}$ isotropic resolution, enabled by image acceleration and undersampled reconstruction techniques, has shown promising results $[15,16]$.

In conventional IR-prepared sequences for LGE imaging there are no provisions for suppressing the signal arising from adipose tissue. Since both myocardial fibrosis and fat have a low T1, they can appear bright in LGE images, hindering the distinction between these tissues. Fat suppression is therefore an additional challenge for 3D LGE imaging, as the presence of fatty infiltration in the myocardium could be mistaken for fibrosis [17]. Furthermore, in applications such as left-atrial LGE imaging, the abundance of epicardial and pericardial fat can obscure the depiction of fibrosis, and is commonly mentioned as a potential confounding factor that can affect the accuracy of the measured fibrosis extent [18-20]. Multi-echo Dixon water/fat separation techniques have been proposed as a solution for improving fat suppression in LGE imaging. This technique has so far been mostly explored in breathheld 2D LGE imaging [21, 22], and more recently in lowresolution single breath-hold 3D LGE imaging [23, 24], showing promising results in terms of depiction of fibrofatty infiltration in the myocardium and better depiction of cardiac masses. Approaches that integrate diaphragmatic navigators with $3 \mathrm{D}$ water/fat LGE imaging to enable free-breathing acquisitions with increased spatial resolution have been demonstrated $[25,26]$, however the use of respiratory gating limited the volumetric coverage and slice thickness when acquired within a clinically acceptable acquisition time.

Another disadvantage of diaphragmatic navigator gating approaches is that they do not measure the respiratory motion of the heart, but infer it from the motion of the diaphragm by assuming a linear correlation between them [27]. However, this simplified model does not account for hysteresis effects or the complex non-rigid motion of the heart during free breathing. In order to address these limitations, respiratory motion compensation techniques based on image navigators (iNAVs) have been introduced for whole-heart CMR imaging. By acquiring lowresolution 2D [28-30] or 3D images [31-34] before or after 3D whole-heart data acquisition, respiratory motion can be directly measured in the heart by tracking a region of interest. iNAV-based approaches incorporate most of the acquired data for image reconstruction ( 95-100\% scan efficiency depending if rejection of outliers is considered), leading to predictable and significantly reduced acquisition times compared to diaphragmatic navigator gating and have been successfully applied to a variety of CMR protocols. Indeed, iNAV-based whole-heart imaging has been integrated into the BOOST framework [35] for simultaneous black-blood LGE and bright-blood coronary 
CMR angiography based on an interleaved $\mathrm{T}_{2}$ preparation ( $\mathrm{T}_{2}$ prep) and $\mathrm{T}_{2}$ prep-IR sequence. A similar approach has been recently proposed for $T_{2}$-prepared water/fat coronary CMR imaging [36], for improved fat saturation at $3 \mathrm{~T}$ based on a dual-echo sequence, where opposed-phase iNAVs were used to estimate respiratory motion. In both studies, however, fully sampled acquisitions were used, leading to long scan times $(\sim 15 \mathrm{~min})$ and a limited spatial resolution. More recently, iNAV-based whole-heart CMR has been applied to IR-prepared 3D LGE imaging [37], however in this study only translational motion of the heart in the superior-inferior and left-right directions was compensated for, and no mechanisms for fat signal suppression were included.

Here we propose a novel framework for highresolution 3D whole-heart water/fat LGE imaging within a clinically feasible scan time of $\sim 8 \mathrm{~min}$. The sequence integrates 2D iNAVs [28] in a dual-echo acquisition for respiratory motion estimation and non-rigid respiratory motion correction [38], enabling $100 \%$ respiratory efficiency (no data rejection) and predictable scan time. In order to further accelerate the scan, an undersampled variable-density Cartesian trajectory with spiral-like profile reordering $[39,40]$ was used together with a recently introduced high-dimensionality patch-based undersampled reconstruction technique (HD-PROST) [41] that exploits local (within a patch), non-local (between similar patches within a neighborhood) and contrast (between the two different contrasts in the dual-echo images) redundancies. In this study, the feasibility of the proposed 3D water/fat LGE imaging approach was tested in a cohort of 20 patients referred for a CMR examination including assessment of myocardial viability.

\section{Methods \\ Imaging framework}

An electrocardiogram (ECG)-triggered dual-echo 3D IRprepared spoiled gradient echo prototype sequence was implemented as illustrated in Fig. 1 (a detailed pulse sequence diagram can be found in Additional File 1). The dual-echo 3D data are acquired following an undersampled variable-density golden-step Cartesian trajectory with spiral profile order sampling (VD-CASPR) [39, 40], so that one spiral-like interleaf is acquired after the application of the IR pulse in each heartbeat. A lowresolution coronal $2 \mathrm{D}$ dual-echo iNAV is acquired immediately before the 3D dual-echo acquisition by adding spatially-encoded low flip-angle excitation pulses [36].

The image reconstruction scheme combines our previously proposed approach for 3D non-rigid motion correction $[36,38]$ with a recently introduced algorithm for multi-contrast undersampled reconstruction [41], as illustrated in Fig. 2. Briefly, the 2D iNAVs are used to estimate the superior-inferior (SI) and right-left (RL) respiratory motion of the heart during the acquisition, by tracking a rectangular template manually selected around the apex and mid-section of the heart. The SI motion is then used to group the 3D dual-echo data into equally populated respiratory bins, which are corrected for translational motion in both the SI and RL directions by applying a liner phase shift in k-space. Respiratoryresolved 3D opposed-phase images are reconstructed using a soft-gated iterative sensitivity encoding method

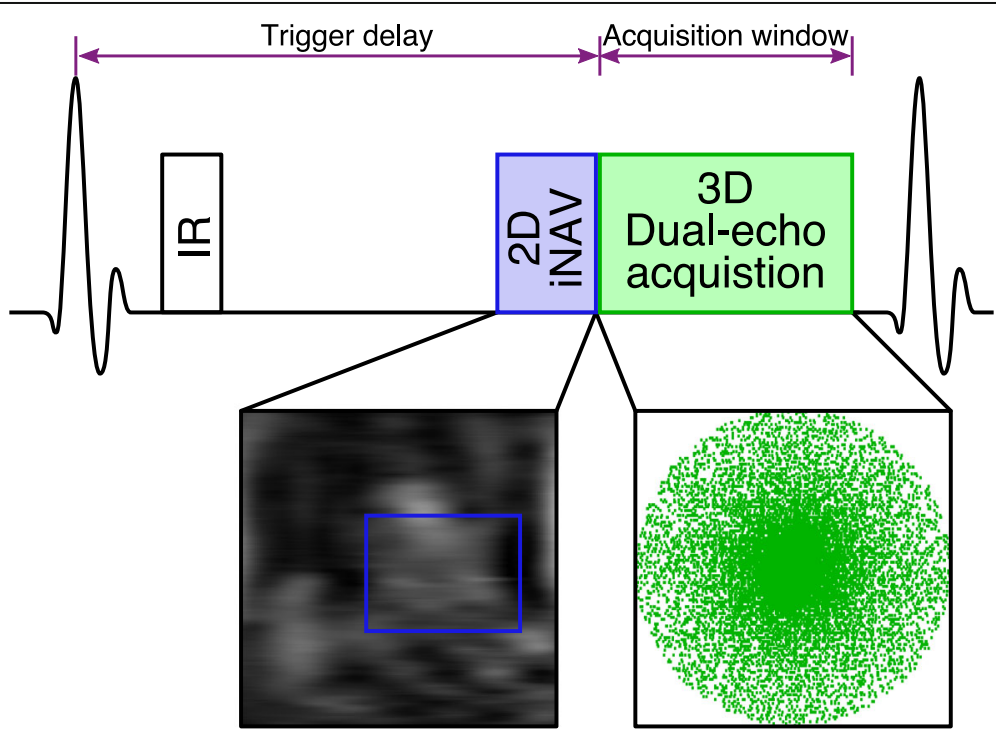

Fig. $13 D$ dual-echo late gadolinium enhancement (LGE) imaging acquisition framework. Low-resolution 2D image-based navigator (iNAVs) are acquired before 3D dual-echo undersampled data acquisition for motion estimation, while inversion recovery (IR) preparation pulses are used to null the viable myocardium signal. The trigger delay and acquisition window are set to coincide with ventricular mid-diastole 


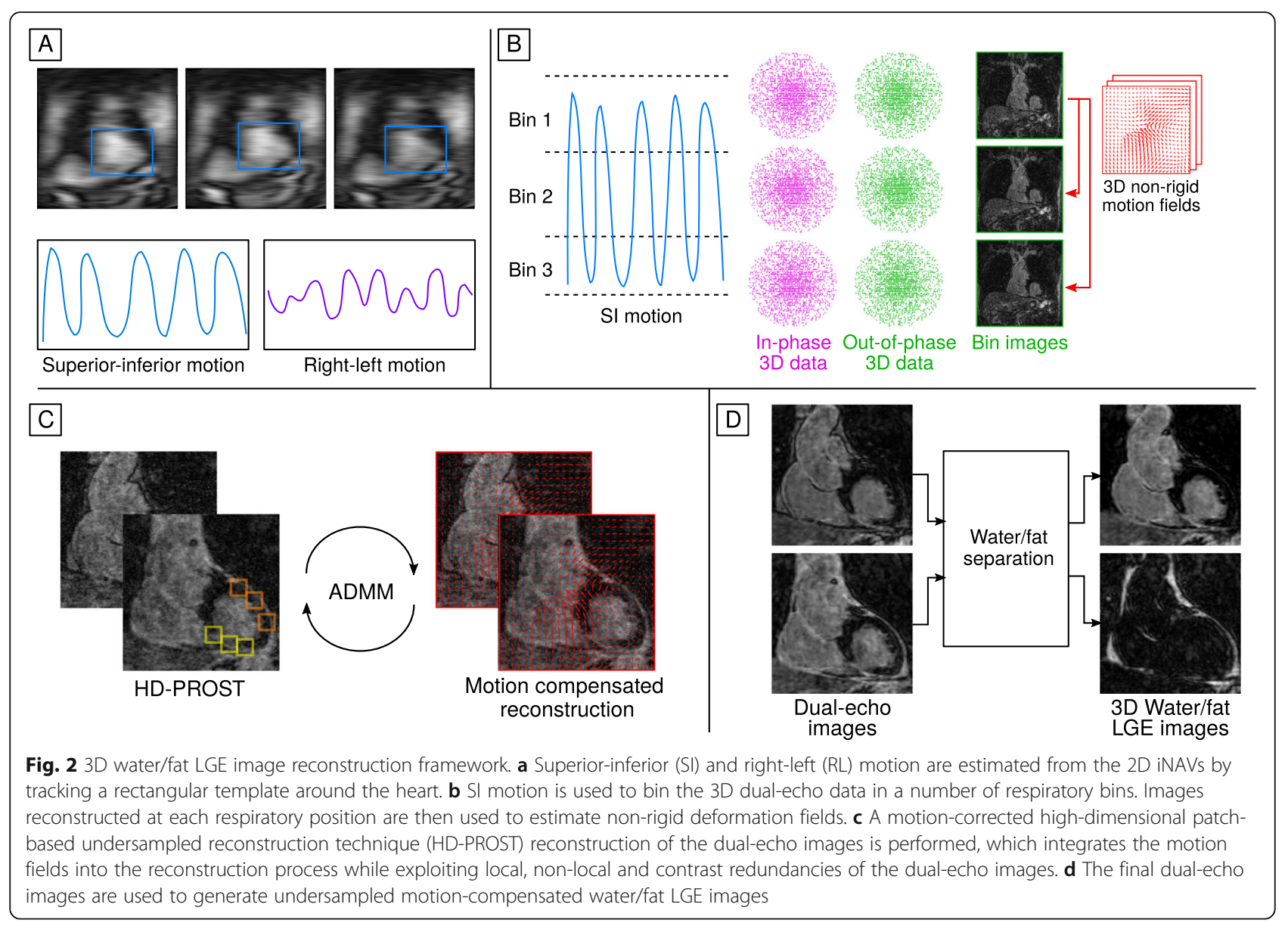

$[38,42]$ and subsequently used to estimate 3D deformation fields via non-rigid image registration using the end-expiration bin as reference.

In order to jointly reconstruct motion-corrected 3D dual-echo images, the non-rigid motion fields are incorporated into an HD-PROST reconstruction, which solves the following problem

$$
\underset{X}{\operatorname{argmin}} \frac{1}{2}\|E X-Y\|_{2}^{2}+\sum_{p} \lambda_{p}\left\|\mathcal{T}_{p}\right\|_{*} \text { s.t. } \mathcal{T}_{p}=R_{p}(X)
$$

where $X$ is the complex 3D dual-echo images to be reconstructed, $E$ is the encoding operator, and $Y$ is the translationally motion-corrected k-space data. The encoding operator $E=\sum S_{b} F U_{b}$ includes the discrete Fourier transform and boil sensitivities, $F$; the non-rigid motion operators $U_{b}$ that transform an image from the reference position to respiratory position $b$; and the sampling matrix $S_{b}$ containing the k-space points acquired at respiratory position $b$. HD-PROST regularizes the image reconstruction problem by representing the multi-contrast (i.e. multi-echo) image $X$ as a low-rank high-order tensor of similar patches $\mathcal{T}_{p}$, whose lowrankness is enforced in the reconstruction by minimizing its nuclear norm $\left\|\mathcal{T}_{p}\right\|_{*}$. $\lambda_{p}$ is the nonnegative regularization parameter and $R_{p}(\cdot)$ is an operator that forms a third order tensor from a patch centered at pixel $p$ from a multi-contrast image. The minimization problem can be solved by alternating direction method of multipliers, as detailed in [41].

Finally, the non-rigid motion-corrected 3D dual-echo images are used to compute the 3D water/fat LGE images by using the B0-NICEbd method [43] for water/fat separation of dual-echo acquisitions performed with bipolar gradients.

\section{Experiments}

The proposed sequence was implemented on a $1.5 \mathrm{~T}$ CMR system (MAGNETOM Aera, Siemens Healthineers, Erlangen, Germany), and all acquisitions were performed on this system using an 18-channel chest-coil and a 32-channel spine coil. Image reconstruction, including motion estimation, non-rigid motioncompensated HD-PROST image reconstruction, and water/fat separation was implemented offline in MATLAB (MathWorks, Inc., Natick, Massachusetts, USA). 
Twenty patients ( 9 male; $57 \pm 16$ years) with known or suspected cardiovascular disease referred for a clinical CMR examination including myocardial viability assessment for both ischemic and non-ischemic cardiomyopathies were recruited between October 2018 and May 2019. Patients were eligible to participate if they were > 18 years of age and agreed to $15 \mathrm{~min}$ of additional CMR imaging after the clinical imaging protocol. The study was approved by the National Research Ethics Service (REC 15/NS/0030). Written informed consent was obtained from each participant according to institutional guidelines. Patient demographics are summarized in Table 1.

The clinical imaging protocol involved a conventional multi-slice 2D LGE acquisition, including 2-chamber, 4chamber and stack of short-axis views $(1.40-1.75 \mathrm{~mm}$ in-plane resolution, $8 \mathrm{~mm}$ slice thickness, 10 to 16 slices covering the left ventricle (LV)). Conventional 2D LGE images were acquired every other heartbeat, starting $10 \mathrm{~min}$ after a bolus administration of $0.15 \mathrm{mmol} / \mathrm{kg}$ of a Gd-based contrast agent (Gadovist, Bayer, Berlin, Germany).

After conventional 2D LGE imaging, the proposed free-breathing 3D water/fat LGE images were acquired with the following parameters: coronal orientation, 3fold undersampling, subject-specific field of view $=312 \times$
$312 \times 83-114 \mathrm{~mm}^{3}$ covering the whole-heart, TR/TE1/ TE2 $=7.16 / 2.38 / 4.76 \mathrm{~ms}$, bipolar gradient readout, receiver bandwidth $=990 \mathrm{~Hz} / \mathrm{px}$, flip angle $=20^{\circ}$, and without additional contrast agent administration. 3D water/ fat LGE data were acquired every heartbeat in 15 patients (indicated as Patients 1 to 15) with an isotropic resolution of $1.3 \mathrm{~mm}^{3}$. Feasibility of performing the acquisition every other heartbeat (as required for patients with very high heart rates and future extension to 3D PSIR water/fat LGE imaging) was investigated in the remaining 5 patients (indicated as Patients 16 to 20). However in this case a resolution of $1.3 \times 1.3 \times 2.6 \mathrm{~mm}^{3}$, interpolated to $1.3 \mathrm{~mm}^{3}$ isotropic during image reconstruction, was adopted to maintain a clinically feasible scan time.

In order to minimize cardiac motion, a subject-specific trigger delay and acquisition window ranging from 105 to $140 \mathrm{~ms}$ (corresponding to 15 to 20 readouts per spiral-like interleaf) were set to coincide with middiastole, by visually inspecting a 4-chamber cine acquisition. The inversion time (TI) was selected to null the signal from viable myocardium by visually inspecting a breath-held 2D TI scout Look-Locker image acquired immediately before the 3D LGE water/fat acquisition, that matched the triggering scheme of the $3 \mathrm{D}$ acquisition (i.e. every heartbeat for Patients 1 to 15, and every

Table 1 Summary of patient demographics

\begin{tabular}{|c|c|c|c|c|}
\hline & Gender & Age & Clinical Condition/Indication & LGE Findings \\
\hline Patient 01 & $\mathrm{~F}$ & 63 & Myocardial infarction & Yes, transmural \\
\hline Patient 02 & M & 51 & Hypertensive cardiomyopathy & No \\
\hline Patient 03 & M & 55 & Suspected ARVC & No \\
\hline Patient 04 & M & 69 & Assessment of inducible ischemia (previous non-STEMI) & No \\
\hline Patient 05 & M & 61 & Suspected DCM (previous atrial fibrillation) & No \\
\hline Patient 06 & $\mathrm{~F}$ & 22 & Myocardial infarction & Yes, near transmural and subendocardial \\
\hline Patient 07 & $\mathrm{~F}$ & 35 & Assessment of angina & No \\
\hline Patient 08 & M & 57 & Assessment of inducible ischemia & No \\
\hline Patient 09 & $\mathrm{~F}$ & 33 & Suspected HCM & Yes, mid-wall \\
\hline Patient 10 & $\mathrm{~F}$ & 55 & Suspected ARVC & No \\
\hline Patient 11 & $\mathrm{~F}$ & 38 & Suspected HCM & No \\
\hline Patient 12 & $\mathrm{~F}$ & 67 & Assessment of cardiac involvement (Hemochromatosis) & No \\
\hline Patient 13 & $\mathrm{~F}$ & 79 & Assessment of inducible ischaemia (previous LBBB) & No \\
\hline Patient 14 & F & 65 & Non-ischemic cardiomyopathy & Yes, mid-wall and subendocardial \\
\hline Patient 15 & F & 86 & Assessment of inducible ischemia (known IHD) & Yes, subendocardial \\
\hline Patient 16 & M & 70 & Myocardial infarction & Yes, transmural and subendocardial \\
\hline Patient 17 & M & 53 & Re-assessment of myocardial fibrosis & Yes, mid-wall and subendocardial \\
\hline Patient 18 & M & 56 & Hypertension & No \\
\hline Patient 19 & M & 50 & Assessment of inducible ischemia (previous DCM) & No \\
\hline Patient 20 & $\mathrm{~F}$ & 71 & Assessment of angina & No \\
\hline
\end{tabular}

Average heart rate for the patients was $65 \pm 13$ beats per minute. ARVC: Arrhythmogenic right ventricular cardiomyopathy; DCM: Dilated cardiomyopathy; HCM: Hypertrophic cardiomyopathy; IHD: Ischemic heart disease; LBBB: Left bundle branch block; STEMI: ST-elevation myocardial infarction 
second heartbeat for Patients 16 to 20). The 2D TI-scout acquisition was performed with a conventional Cartesian trajectory with low-high profile order. The 2D iNAVs were acquired using the following parameters: same field of view as the $3 \mathrm{D}$ water/fat LGE acquisition, flip-angle = $3^{\circ}, 14$ readouts acquired with a high-low Cartesian trajectory, corresponding to a $1.3 \times 22.3 \mathrm{~mm}^{2}$ acquired resolution, reconstructed to $1.3 \times 1.3 \mathrm{~mm}^{2}$ for estimation of SI and RL translational motion.

\section{Image analysis}

Reconstructed 3D water/fat LGE magnitude images were written into digital imaging and communications in medicine (DICOM) format before image quality assessment. For all datasets, presence of water/fat swaps in the 3D LGE images was visually assessed. In order to compare image quality and detectability of fibrosis/scar, 3D water LGE images and conventional magnitude 2D LGE images were considered. For the conventional 2D LGE, magnitude images were available from the scanner vendor software. Images considered for image analysis included 2-chamber, 4-chamber and a stack of shortaxis views. Qualitative grading of the images was performed by one cardiologist with 14 years of experience in CMR imaging (P.G.M.), who was blinded to patient information and history. Both 2D and 3D LGE images were imported to Osirix (Pixmeo, Bernex, Switzerland) for assessment, so that brightness/contrast could be freely adjusted, and for the 3D images both original and multi-planar reformatted images were considered for analysis.

For each dataset, presence of fibrosis/scar was assessed with a 3-point scale, where 0 : Absent with confidence, 1 : Present with confidence, 2: Unable to interpret/inconclusive. Furthermore, the images were graded in terms of image quality using a 4-point scale, with 1: Full diagnostic, 2: Good, 3: Acceptable, 4: Non-diagnostic. This assessment considered the following criteria: ability to visualize LV myocardium, presence of residual respiratory motion and blurring, contrast between scar and viable myocardium, nulling point of the viable myocardium and presence of fold over artifacts. Image quality scores were compared with a paired Wilcoxon signed-rank test to assess statistical differences; $p<0.05$ was considered statistically significant.

Regions of interest (ROIs) were manually drawn in LV blood pool, viable myocardium and fibrotic tissue (when present) at matching locations in order to compute scarto-myocardium, scar-to-blood and blood-tomyocardium contrast ratio in both 2D and 3D LGE images. Contrast ratio was then compared using a paired 2-tailed Student t-test to assess statistical differences with $p<0.05$ considered statistically significant.
Acquisition time was recorded for all scans, including pauses between breath-holds for conventional 2D LGE imaging.

\section{Results}

The proposed 3D water/fat LGE acquisition was successfully completed in all subjects; with an average scan time of $8.0 \pm 1.4 \mathrm{~min}$, and data acquisition starting $25.9 \pm 7.7$ min after contrast administration. The conventional $2 \mathrm{D}$ LGE datasets required a statistically significant $(p<$ 0.001 ) shorter scan time of $5.6 \pm 2.2 \mathrm{~min}$, including pauses between breath holds. Water/fat swaps were observed in two patients. In one patient, local water/fat swaps were observed in breast implants, without affecting the water/fat separation elsewhere in the field of view. In the second patient, a global water/fat swap was observed, i.e. the water image was mislabeled as fat image. In both cases, the presence of swaps did not affect image quality around the heart.

Image quality was considered diagnostic (graded in categories 1 to 3 ) in 18 out of 20 cases, for both 2D LGE and $3 \mathrm{D}$ water LGE images. Two cases were graded as 4, i.e. non-diagnostic in both the 2D and 3D LGE images, one due to the presence of magnetic field inhomogeneities that resulted in pronounced artifacts in the images (Patient 13), and one due to the presence of residual cardiac motion and wrong nulling point of the myocardium (Patient 20). These two cases were therefore excluded from the following analysis. Image quality scores for the remaining patients $(n=18)$ were $2.05 \pm 0.72$ for the $2 \mathrm{D}$ LGE images and $1.88 \pm 0.90$ for the 3D water LGE images. No statistically significant difference was found between the image quality scores in both protocols $(p=$ 0.62).

In 11 patients, LGE was deemed as absent in both the 2D LGE and the 3D water LGE images, while in 5 cases there was agreement about the presence of LGE between the two techniques. In two further cases, presence of LGE was found with confidence in 3D water LGE images, however interpretation of the corresponding $2 \mathrm{D}$ LGE images was inconclusive. A summary of the qualitative analysis can be found in Table 2 .

In conventional 2D LGE images, scar-to-myocardium contrast ratio was $7.46 \pm 2.61$ on average, while bloodto-myocardium and scar-to-blood contrast ratio were $6.7 \pm 2.7$ and $0.0 \pm 0.2$ respectively. For the water $3 \mathrm{D}$ LGE images, scar-to-myocardium contrast ratio was $7.3 \pm 4.6$ on average, with no statistically significant difference found between 2D and 3D images $(p=0.87)$. Blood-to-myocardium contrast ratio was $3.3 \pm 1.8$ for the $3 \mathrm{D}$ images, significantly lower than in the $2 \mathrm{D}$ images $(p<0.001)$, while scar-to-blood contrast ratio resulted significantly higher $(0.6 \pm 0.7, p=0.03)$. 
Table 2 Summary of image quality assessment

\begin{tabular}{lcccc}
\hline & Image & \multicolumn{3}{c}{ Diagnostic outcome } \\
\cline { 3 - 5 } & Quality & LGE Present & LGE Absent & Inconclusive \\
\hline 2D magnitude & $2.05 \pm 0.72$ & 5 & 11 & 2 \\
3D Dixon - water & $1.88 \pm 0.90$ & 7 & 11 & 0
\end{tabular}

Summary of image quality assessment for conventional 2D magnitude and 3D water LGE images in 18/20 cases deemed diagnostic. Image quality scores: 1 : Full diagnostic, 2: Good, 3: Acceptable, 4: Non-diagnostic

A multi-planar reformatting of a 3D water LGE image acquired every heartbeat for a representative patient (Patient 7) is shown in Fig. 3, including vertical and horizontal long axis and short axis views. An improved depiction of small features compared to conventional 2D LGE images, such as the papillary muscles, can be observed with $3 \mathrm{D}$ water LGE (a visual comparison of 2D and 3D LGE images for Patient 7 can be found in Additional File 2).

A comparison between 3D water LGE images acquired every heartbeat and 2D LGE images is shown in Fig. 4, including a short axis and vertical long axis view for two additional patients. Small areas of LGE can be observed in Patient 6, both in the conventional 2D LGE and the 3D water LGE horizontal long axis view images (green arrows), with the $3 \mathrm{D}$ images showing an improved delineation of the areas of enhancement in the short axis view. In Patient 8 , a similar depiction of the LV myocardium can be observed in both 2D and 3D LGE images.

A coronal slice and a short-axis view showing water and fat images for three additional patients are shown in Fig. 5. In particular, in Patient 1 the basal transmural infarction (red arrow) is well delineated even in presence of pericardial fat thanks to the water/fat separation approach.

Similar results were obtained for the datasets acquired every other heartbeat, as can be observed in Fig. 6, which shows a multi-planar reformatting of the 3D water LGE image alongside conventional 2D LGE images for Patient 16. A clear depiction of a small sub-endocardial infarction in the anterior wall (red arrow) can be observed in the whole-heart 3D LGE image (see Additional File 3 for a complete whole-heart multiplanar reformatting), which is consistent with findings observed in the conventional $2 \mathrm{D}$ LGE protocol (red arrow). Figure 7 shows a further visual comparison between 3D water/fat LGE images acquired every other heartbeat and 2D LGE images

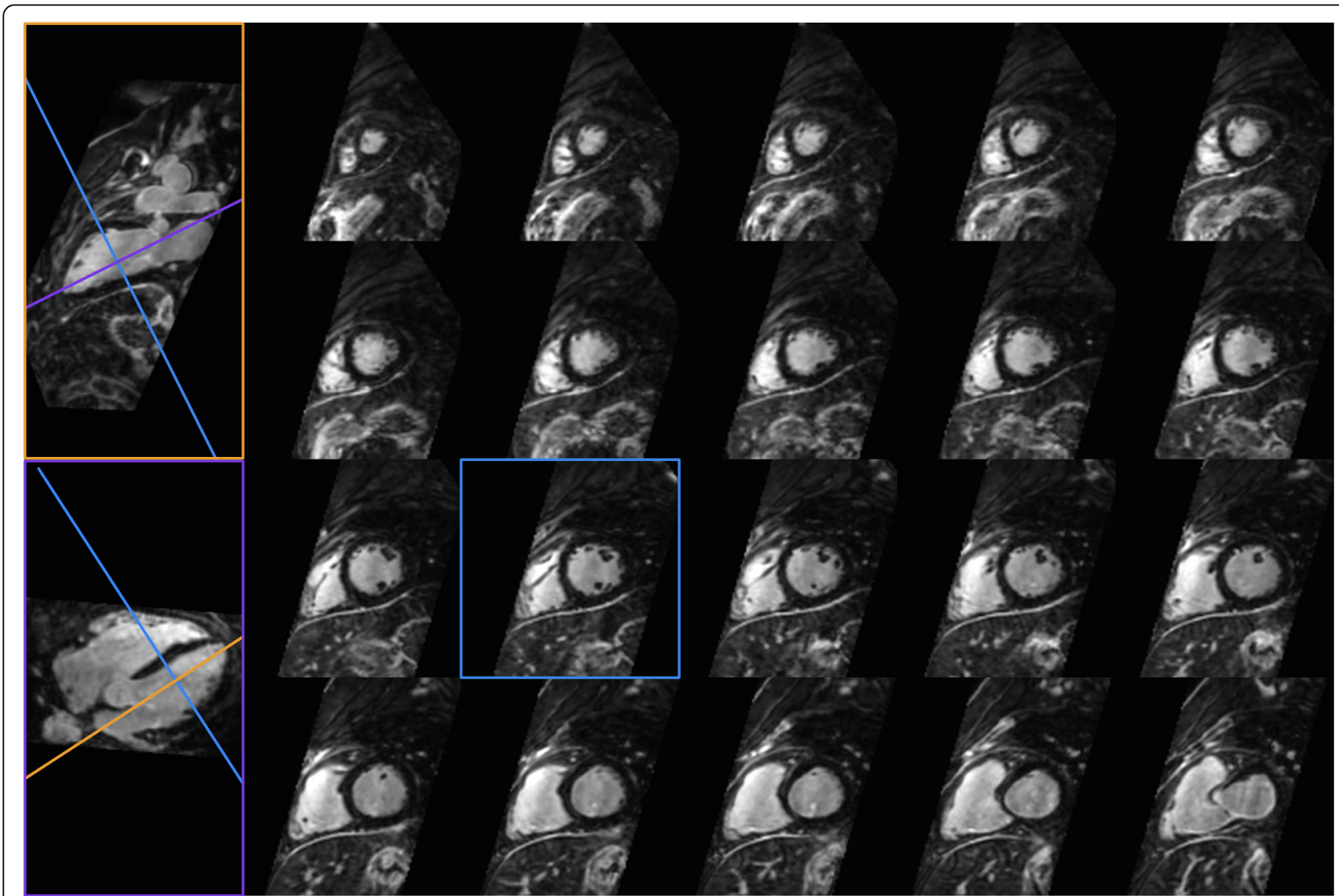

Fig. 3 Multi-planar reformatting of the 3D (water) LGE image acquired every heartbeat for Patient 7, showing vertical and horizontal long axis and short axis views. The high isotropic acquired resolution enables the depiction of small features, such as the papillary muscles 


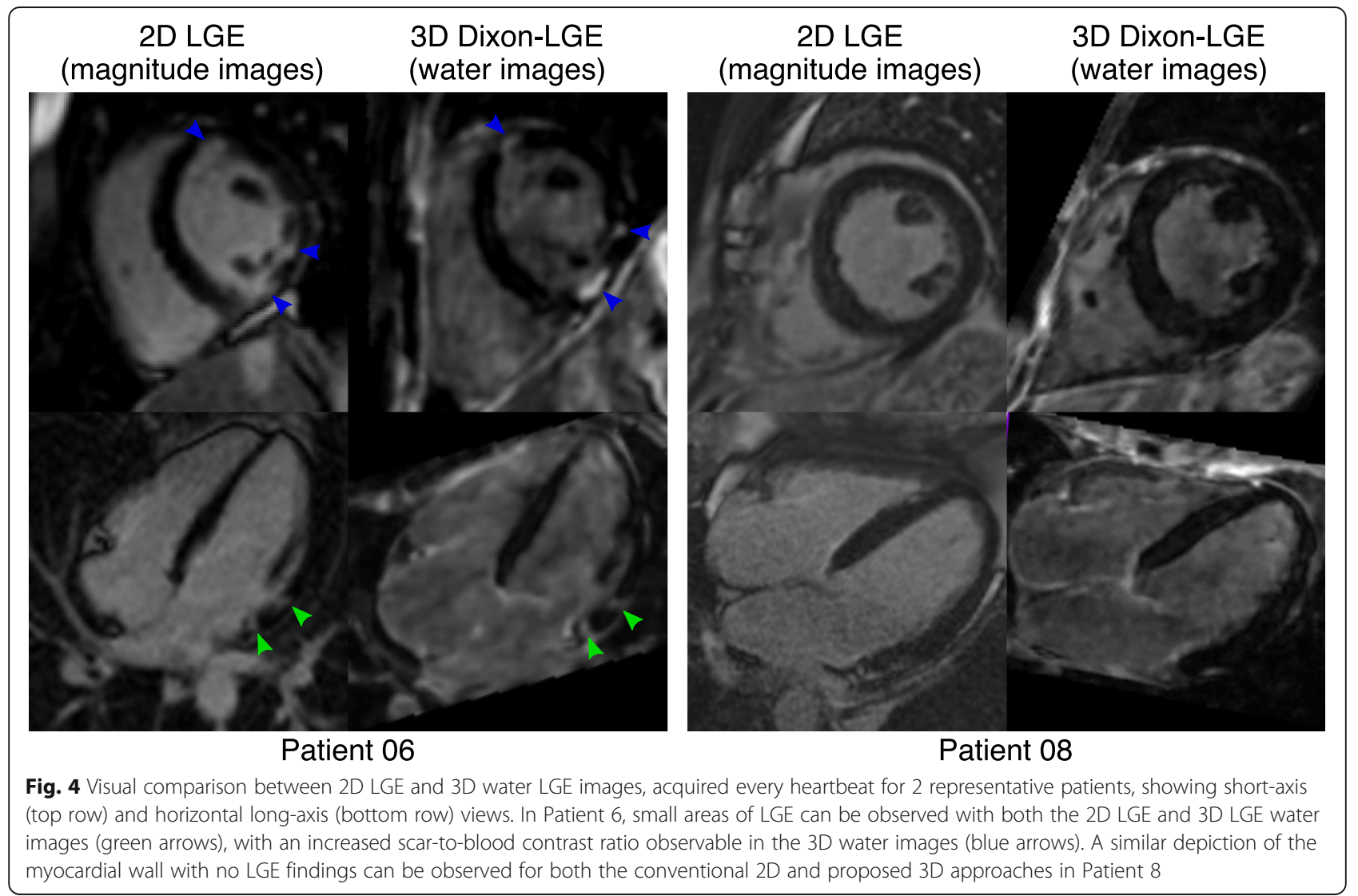

for two representative patients, including short axis and horizontal long axis view. In Patient 17, a comparable depiction of the myocardial wall and scar can be observed (green arrows) between both scans, while in Patient 18 the effect of complete fat suppression in the 3D water LGE images is apparent (blue arrows).

\section{Discussion}

In this study we demonstrate the feasibility of a novel framework for motion corrected high-resolution 3D water/fat LGE imaging. Compared to previously proposed approaches for free-breathing 3D water/fat LGE imaging $[25,26]$ that rely on diaphragmatic navigator

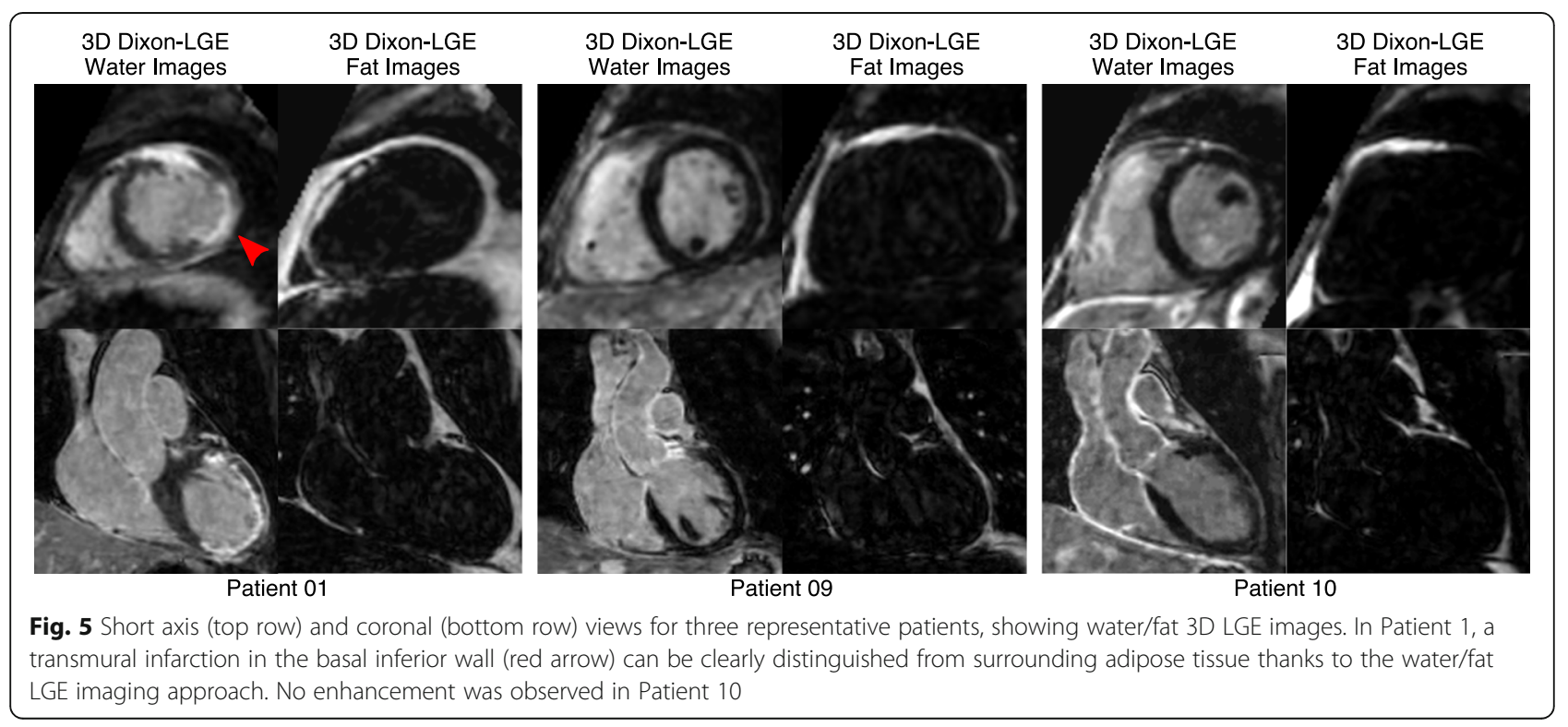



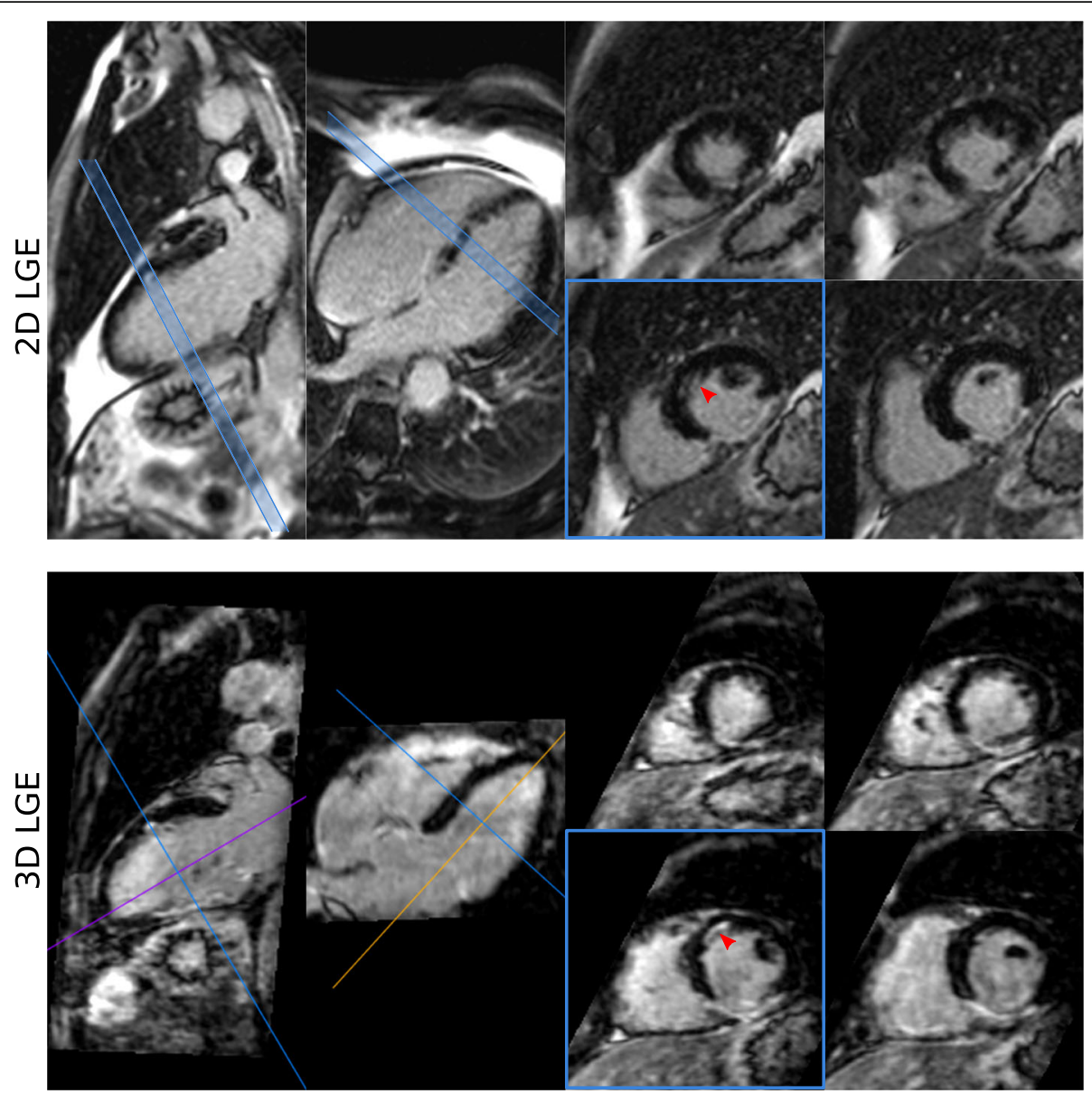

Fig. 6 Visual comparison between conventional 2D LGE and proposed 3D LGE images acquired for Patient 16, showing vertical and horizontal long axis, and four short axis slices acquired for the left ventricle myocardium. The small subendocardial infarction can be observed in the midanterior wall (red arrow) in both sets of images, with a better depiction in the long axis in the case of the 2D LGE images; and a good depiction in both long and short axis views in the 3D LGE images

gating to compensate for respiratory motion, our framework makes use of $2 \mathrm{D}$ iNAVs to directly track the respiratory motion of the heart, enabling 100\% respiratory scan efficiency and a predictable scan time that depends only on the subjects' heart rate and the volumetric coverage required. Consequently, for a given scan time, the iNAV-based motion correction approach can achieve a higher spatial resolution compared to diaphragmatic navigator gating approaches. Furthermore, compared to a recently introduced approach for iNAV-based 3D LGE imaging [37], which corrects for translational motion of the heart in SI and RL directions only, and to a recently proposed approach based on three one-dimensional navigators for 3D translational motion-corrected 3D LGE imaging [44], our approach corrects for the complex non-rigid motion of the heart during free breathing and enables a higher spatial resolution by integrating an undersampled acquisition trajectory.

3D water LGE images were graded as diagnostic in $18 / 20$ cases and image quality scores were similar $(p=0.62)$ when comparing conventional breath held $2 \mathrm{D}$ and the proposed 3D water/fat LGE imaging technique. In most cases (16/18 diagnostic cases), there was good agreement between the $3 \mathrm{D}$ and the $2 \mathrm{D}$ technique in terms of presence of LGE findings. In two further cases the 2D LGE images were inconclusive while the 3D water images indicated presence of LGE findings, with the assessment facilitated by an increased spatial resolution, increased contrast ratio between scar and blood pool and/or improved fat suppression. Whereas some of these improvements can be attributed to an increased scar-to-blood contrast ratio in the 3D LGE images, likely due to the washout of contrast agent (e.g. in Patient 06, Fig. 4), in other cases the increased resolution and reduced slice thickness of the 3D LGE images potentially enabled improved depiction of small scar (e.g. in Patient 16, Fig. 6).

The high-resolution whole-heart 3D water LGE images obtained with the proposed approach enable multi- 


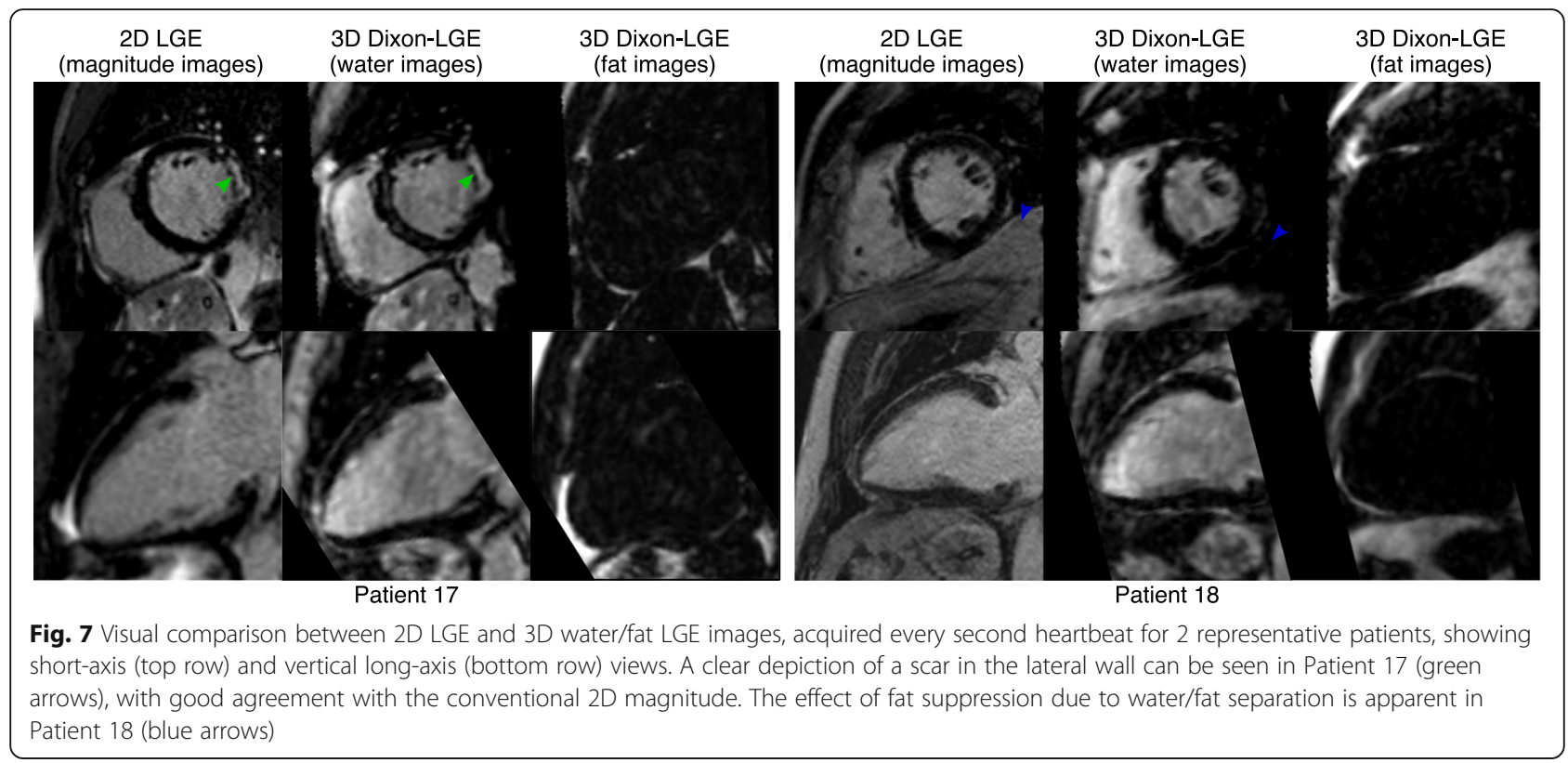

planar reformatting in any direction. The presence of small areas of LGE can be assessed in such images without the need of performing additional acquisitions in oblique planes, as it is often the case in conventional breath held 2D LGE imaging. Therefore, the ability of the proposed framework to produce high-resolution 3D images might be of clinical relevance for the accurate depiction of sub-endocardial fibrosis, or in clinical applications that focus on smaller cardiac structures, such as in atrial wall LGE imaging. Nevertheless, further clinical studies are required to evaluate the clinical impact of increasing spatial resolution in LGE imaging.

The 3D water/fat LGE imaging method was demonstrated for acquisitions every heartbeat with high isotropic resolution and every other heartbeat with an increased slice thickness, with good image quality obtained in both cases. In the clinical routine, either approach could be used depending on the heart rate of the subject being scanned [45].

While in this study all acquisitions were performed in coronal orientation, the same approach can be extended to other orientations, and used for instance in clinical applications that favor axial acquisitions [46]. Furthermore, as the motion-compensated HD-PROST reconstruction approach provides non-rigid respiratory motion fields, the method could be extended to $3 \mathrm{~T}$ hybrid positron emission tomography (PET)-CMR systems for simultaneous motion-corrected cardiac PET-CMR imaging. Such an approach would be of interest for producing co-registered truly simultaneous images of myocardial inflammation by ${ }^{18} \mathrm{~F}$-fluorodeoxyglucose-PET and myocardial viability by 3D LGE-CMR imaging, aiding the interpretation of findings from both imaging modalities and potentially improving the diagnosis of conditions such as myocarditis and cardiac sarcoidosis [47-50].

\section{Limitations}

This study has some limitations. While the effect of respiratory motion was addressed by the motion-corrected HD-PROST reconstruction, residual cardiac motion can be present in the 3D water/fat LGE images in patients with heart rate variability. Furthermore, in cases of erratic breathing patterns, the image quality of respiratory bins might be insufficient to produce accurate non-rigid motion estimates. Future work will include the integration of mechanisms for erratic respiratory outlier rejection and arrhythmia rejection, which may further improve image quality. Although a dedicated TI scout was performed before 3D water/fat LGE acquisition to select the optimal inversion time for myocardial nulling, this time changes over the $\sim 8 \mathrm{~min}$ acquisition (due to contrast agent washout), resulting in some cases in suboptimal contrast between scar and myocardium. Further acceleration to reduce scan time could alleviate this problem, by both reducing the time for contrast agent washout and potentially enabling the extension of the framework to PSIR imaging. Alternatively, a change in the administration of the contrast agent from a bolus injection to slow infusion could further improve image quality and robustness of the technique.

This study compared image quality and agreement in LGE findings between conventional 2D magnitude LGE and the proposed 3D water LGE approaches in a small cohort of patients. However, as the study recruited patients willing to undertake an additional research scan 
immediately after a clinical referred examination, the 3D water/fat LGE data needed to be performed $\sim 15 \mathrm{~min}$ after the start of the clinical 2D LGE examination. Furthermore, the study recruited a limited number of patients with LGE findings. Further studies in a larger cohort of patients with known chronic myocardial infarction, and with the order of the acquisition of the two techniques being randomized are required to fully characterize the performance of the proposed method, including a comprehensive analysis of the lesion detectability for various lesion sizes for both 2D and 3D LGE images.

The proposed method provides a complementary fat image that could be valuable for a comprehensive assessment of fibro-fatty infiltration in the myocardium. However, cases of fatty infiltration of the myocardium were not observed in the patient cohort recruited for this study, and thus further studies to investigate the clinical value of the whole-heart fat images are needed.

Finally, in this study the image reconstruction pipeline was implemented offline. Future work will include implementation of the motion-compensated HD-PROST method in the scanner software to facilitate clinical translation of the technique.

\section{Conclusions}

A novel framework for undersampled free-breathing high-resolution 3D whole-heart water/fat LGE imaging has been presented in this study. The proposed acquisition approach is highly efficient, as it incorporates all the acquired data in the reconstruction process (no data rejection), enabling a predictable and overall short scan duration of $\sim 8 \mathrm{~min}$.

The framework was demonstrated in a group of patients with known or suspected cardiovascular disease. The resulting 3D water LGE images showed good quality depiction of myocardial fibrosis/scar and image quality comparable to conventional 2D LGE magnitude images, while offering a superior whole-heart coverage and higher spatial resolution.

\section{Supplementary information}

Supplementary information accompanies this paper at https://doi.org/10. 1186/s12968-020-00649-5.

Additional file 1. Pulse sequence diagram, showing one RF excitation (in black) and the corresponding dual-echo readout gradient (in blue), indicating echo times TE1 and TE2. The intervals where data are acquired are indicated with a green box.

Additional file 2. Visual comparison between conventional 2D LGE and proposed 3D LGE images acquired for Patient 7, showing vertical and horizontal long axis, and four short axis slices acquired for the left ventricle myocardium. The high spatial resolution of the 3D LGE images enables a good depiction of small features such as the papillary muscles.

Additional file 3. Multi-planar reformatting of the 3D (water) LGE image acquired every second heartbeat for Patient 16 , showing vertical and horizontal long axis and short axis views. The spatial resolution of the images enables a clear depiction of a small anterior sub-endocardial myocardial infarction in the short axis view (red arrow).

\begin{abstract}
Abbreviations
ARVC: Arrhythmogenic ventricular cardiomyopathy; CMR: Cardiovascular magnetic resonance; DCM: Dilated cardiomyopathy; DICOM: Digital imaging and communications in medicine; ECG: Electrocardiogram;

HCM: Hypertrophic cardiomyopathy; HD-PROST: High-dimensional patchbased undersampled reconstruction; ICM: Ischemic cardiomyopathy; iNAV: Image navigator; IR: Inversion recovery; LBBB: Left bundle branch block; LGE: Late gadolinium enhancement; LV: Left ventricle/left ventricular; PET: Positron emission tomography; PSIR: Phase-sensitive inversion recovery; RL: Right-left; ROI: Region of interest; SI: Superior-inferior; STEMI: ST elevation myocardial infarction; T2prep: T2 prepared; TI: Inversion time; VD-

CASPR: variable-density golden-step Cartesian trajectory with spiral profile order sampling
\end{abstract}

\section{Acknowledgements}

This work was supported by the following grants: EPSRC 1) EP/P032311/1; 2) EP/P007619/1; 3) EP/P001009/1; and the Wellcome/EPSRC Centre for Medical Engineering (WT 203148/Z/16/Z). This research was supported by the National Institute for Health Research (NIHR) Cardiovascular Health

Technology Cooperative (HTC) and the Biomedical Research Centre based at Guy's and St. Thomas' NHS Foundation Trust and King's College London.

\section{JCMR guest editor}

Dr. Robert Judd served as a JCMR Guest Editor for this manuscript.

\section{Authors' contributions}

$C M, M Z, W W, R B$ and $C P$ contributed to the study design. $C M, A B, R N, K K, C F$ and $M S$ contributed to the implementation of the acquisition sequence. $C M$, $A B, R B$ and $C P$ contributed to the design and implementation of the reconstruction and motion correction framework. $\mathrm{CM}$ and $\mathrm{RH}$ performed the acquisition of the clinical research data. PGM performed the image quality evaluation. CM and PGM performed the data analysis. All the authors participated in drafting and revising the manuscript, and read and approved its final version.

\section{Availability of data and materials}

Anonymized DICOM images from patient acquisitions are not publicly available but are available from the corresponding author upon reasonable request.

\section{Ethics approval and consent to participate}

This study was approved by the National Research Ethics Service (REC 15/NS/ 0030). Written informed consent was obtained for all the subjects. Anonymized data were analyzed at the School of Biomedical Engineering and Imaging Sciences (King's College London) at St. Thomas' Hospital.

\section{Consent for publication}

All the subjects provided written informed consent for the publication of accompanying images in this manuscript. The consent forms are held in the patients' clinical notes and are available to the Editor-in-Chief upon request.

\section{Competing interests}

K.K., R.N., C.F., M.S. are employed by Siemens Healthcare. All other authors declare that they do not have competing interests.

\section{Author details}

${ }^{1}$ School of Biomedical Engineering and Imaging Sciences, King's College London, St Thomas' Hospital, 3rd Floor, Lambeth Wing, London SE1 7EH, UK. ${ }^{2}$ MR Research Collaborations, Siemens Healthcare, Frimley, UK.

${ }^{3}$ Cardiovascular MR Predevelopment, Siemens Healthcare GmbH, Erlangen, Germany. ${ }^{4}$ Institute of Radiology, University Hospital Erlangen, Erlangen, Germany. 
Received: 3 October 2019 Accepted: 17 June 2020 Published online: 20 July 2020

\section{References}

1. Kim RJ, Fieno DS, Parrish TB, et al. Relationship of MRI delayed contrast enhancement to irreversible injury, infarct age, and contractile function. Circulation. 1999;100:1992-2002. https://doi.org/10.1161/01.CIR.100.19.1992.

2. Simonetti OP, Kim RJ, Fieno DS, et al. An improved MR imaging technique for the visualization of myocardial infarction. Radiology. 2001;218:215-23. https://doi.org/10.1148/radiology.218.1.r01ja50215.

3. Kellman P, Arai AE. Cardiac imaging techniques for physicians: late enhancement. J Magn Reson Imaging. 2012;36:529-42. https://doi.org/10. 1002/jmri.23605

4. Kellman $P$, Arai $A E, M c V e i g h ~ E R$, Aletras $A H$. Phase-sensitive inversion recovery for detecting myocardial infarction using gadolinium-delayed hyperenhancement. Magn Reson Med. 2002;47:372-83. https://doi.org/10. 1002/mrm.10051.

5. Kramer CM, Barkhausen J, Flamm SD, Kim RJ, Nagel E. Standardized cardiovascular magnetic resonance (CMR) protocols 2013 update. J Cardiovasc Magn Reson. 2013;15:91. https://doi.org/10.1186/1532-429X-1591.

6. Kramer CM, Barkhausen J, Bucciarelli-Ducci C, Flamm SD, Kim RJ, Nagel E. Standardized cardiovascular magnetic resonance imaging (CMR) protocols: 2020 update. J Cardiovasc Magn Reson. 2020;22:17. https://doi.org/10.1186/ s12968-020-00607-1.

7. Saranathan M, Rochitte CE, Foo TKF. Fast, three-dimensional free-breathing MR imaging of myocardial infarction: a feasibility study. Magn Reson Med. 2004;51:1055-60. https://doi.org/10.1002/mrm.20061.

8. Peters DC, Wylie JV, Hauser TH, et al. Detection of pulmonary vein and left atrial scar after catheter ablation with three-dimensional navigator-gated delayed enhancement MR imaging: initial experience. Radiology. 2007;243: 690-5. https://doi.org/10.1148/radiol.2433060417.

9. Amano $Y$, Matsumura $Y$, Kumita $S$. Free-breathing high-spatial-resolution delayed contrast-enhanced three-dimensional viability MR imaging of the myocardium at 3.0T: a feasibility study. J Magn Reson Imaging. 2008;28: 1361-7. https://doi.org/10.1002/jmri.21595.

10. Nguyen TD, Spincemaille $P$, Weinsaft JW, et al. A fast navigator-gated $3 D$ sequence for delayed enhancement MRI of the myocardium: comparison with breathhold 2D imaging. J Magn Reson Imaging. 2008;27:802-8. https:// doi.org/10.1002/jmri.21296.

11. Peters DC, Appelbaum EA, Nezafat R, et al. Left ventricular infarct size, periinfarct zone, and papillary scar measurements: a comparison of highresolution $3 \mathrm{D}$ and conventional $2 \mathrm{D}$ late gadolinium enhancement cardiac MR. J Magn Reson Imaging. 2009;30:794-800. https://doi.org/10.1002/jmri. 21897.

12. Kino A, Zuehlsdorff S, Sheehan JJ, et al. Three-dimensional phase-sensitive inversion-recovery turbo FLASH sequence for the evaluation of left ventricular myocardial scar. Am J Roentgenol. 2009;193:W381-8. https://doi. org/10.2214/AJR.08.1952.

13. Peters DC, Shaw JL, Knowles BR, Moghari MH, Manning WJ. Respiratory bellows-gated late gadolinium enhancement of the left atrium. J Magn Reson Imaging. 2013;38:1210-4. https://doi.org/10.1002/jmri.23954.

14. Keegan J, Drivas P, Firmin DN. Navigator artifact reduction in threedimensional late gadolinium enhancement imaging of the atria. Magn Reson Med. 2014;72:779-85. https://doi.org/10.1002/mrm.24967.

15. Akçakaya M, Rayatzadeh H, Basha TA, et al. Accelerated late gadolinium enhancement cardiac MR imaging with isotropic spatial resolution using compressed sensing: initial experience. Radiology. 2012;264:691-9. https:// doi.org/10.1148/radiol.12112489.

16. Basha TA, Akçakaya M, Liew C, et al. Clinical performance of high-resolution late gadolinium enhancement imaging with compressed sensing. J Magn Reson Imaging. 2017;46:1829-38. https://doi.org/10.1002/jmri.25695.

17. Kellman P, Hernando D, Arai AE. Myocardial fat imaging. Curr CardiovasC Imaging Rep. 2010;3:83-91. https://doi.org/10.1007/s12410-010-9012-1.

18. Akkaya M, Hiquchi $K$, Koopmann M, et al. Relationship between left atrial tissue structural remodelling detected using late gadolinium enhancement MRI and left ventricular hypertrophy in patients with atrial fibrillation. Europace. 2013;15:1725-32. https://doi.org/10.1093/europace/eut147.

19. Dewire J, Khurram IM, Pashakhanloo F, et al. The association of pre-existing left atrial fibrosis with clinical variables in patients referred for catheter ablation of atrial fibrillation. Clin Med Insights Cardiol. 2014;8:25-30. https:// doi.org/10.4137/CMC.S15036.

20. Habibi M, Lima JAC, Khurram IM, et al. Association of left atrial function and left atrial enhancement in patients with atrial fibrillation cardiac magnetic resonance study. Circ Cardiovasc Imaging. 2015;8:e002769. https://doi.org/ 10.1161/CIRCIMAGING.114.002769.

21. Goldfarb JW. Fat-water separated delayed hyperenhanced myocardial infarct imaging. Magn Reson Med. 2008;60:503-9. https://doi.org/10.1002/ mrm.21685.

22. Kellman P, Hernando D, Shah S, et al. Multiecho Dixon fat and water separation method for detecting fibrofatty infiltration in the myocardium. Magn Reson Med. 2009;61:215-21. https://doi.org/10.1002/mrm.21657.

23. Saranathan M, Glockner J. Three-dimensional Dixon fat-water separated rapid breathheld imaging of myocardial infarction. J Magn Reson Imaging. 2013:38:1362-8. https://doi.org/10.1002/jmri.24113.

24. Foley JRJ, Fent GJ, Garg P, et al. Feasibility study of a single breath-hold, 3D mDIXON pulse sequence for late gadolinium enhancement imaging of ischemic scar. J Magn Reson Imaging. 2019;49:1437-45. https://doi.org/10. 1002/jmri.26519.

25. Havla L, Basha T, Rayatzadeh H, et al. Improved fat water separation with water selective inversion pulse for inversion recovery imaging in cardiac MRI. J Magn Reson Imaging. 2013;37:484-90. https://doi.org/10.1002/jmri. 23779.

26. Shaw JL, Knowles BR, Goldfarb JW, Manning WJ, Peters DC. Left atrial late gadolinium enhancement with water-fat separation: the importance of phase-encoding order. J Magn Reson Imaging. 2014;40:119-25. https://doi. org/10.1002/jmri.24340.

27. Wang Y, Riederer SJ, Ehman RL. Respiratory motion of the heart: kinematics and the implications for the spatial resolution in coronary imaging. Magn Reson Med. 1995:33:713-9. https://doi.org/10.1002/mrm.1910330517.

28. Henningsson M, Koken P, Stehning C, Razavi R, Prieto C, Botnar RM. Wholeheart coronary MR angiography with 2D self-navigated image reconstruction. Magn Reson Med. 2012;67:437-45. https://doi.org/10.1002/ mrm.23027.

29. Wu HH, Gurney PT, Hu BS, Nishimura DG, McConnell MV. Free-breathing multiphase whole-heart coronary MR angiography using image-based navigators and three-dimensional cones imaging. Magn Reson Med. 2013; 69:1083-93. https://doi.org/10.1002/mrm.24346.

30. Kawaji K, Spincemaille P, Nguyen TD, et al. Direct coronary motion extraction from a 2D fat image navigator for prospectively gated coronary MR angiography. Magn Reson Med. 2014;71:599-607. https://doi.org/10. 1002/mrm.24698.

31. Keegan J, Gatehouse PD, Yang GZ, Firmin DN. Non-model-based correction of respiratory motion using beat-to-beat 3D spiral fat-selective imaging. J Magn Reson Imaging. 2007;26:624-9. https://doi.org/10.1002/jmri.20941.

32. Scott AD, Keegan J, Firmin DN. Beat-to-beat respiratory motion correction with near 100\% efficiency: a quantitative assessment using high-resolution coronary artery imaging. Magn Reson Imaging. 2011;29:568-78. https://doi. org/10.1016/j.mri.2010.11.004

33. Moghari MH, Roujol S, Henningsson M, et al. Three-dimensional heart locator for whole-heart coronary magnetic resonance angiography. Magn Reson Med. 2014;71:2118-26. https://doi.org/10.1002/mrm.24881.

34. Addy NO, Ingle RR, Luo J, et al. 3D image-based navigators for coronary MR angiography. Magn Reson Med. 2017;77:1874-83. https://doi.org/10.1002/ mrm.26269.

35. Ginami G, Neji R, Rashid I, et al. 3D whole-heart phase sensitive inversion recovery CMR for simultaneous black-blood late gadolinium enhancement and bright-blood coronary CMR angiography. J Cardiovasc Magn Reson. 2017;19:94. https://doi.org/10.1186/s12968-017-0405-z.

36. Munoz C, Cruz G, Neji R, Botnar RM, Prieto C. Motion corrected water/fat whole-heart coronary MR angiography with $100 \%$ respiratory efficiency. Magn Reson Med. 2019;82:732-42. https://doi.org/10.1002/mrm.27732.

37. Bratis K, Henningsson M, Grigoratos C, et al. Image-navigated 3-dimensional late gadolinium enhancement cardiovascular magnetic resonance imaging: feasibility and initial clinical results. J Cardiovasc Magn Reson. 2017;19:97. https://doi.org/10.1186/s12968-017-0418-7.

38. Cruz G, Atkinson D, Henningsson M, Botnar RM, Prieto C. Highly efficient nonrigid motion-corrected 3D whole-heart coronary vessel wall imaging. Magn Reson Med. 2017;77:1894-908. https://doi.org/10.1002/mrm.26274.

39. Prieto $C$, Doneva $M$, Usman $M$, et al. Highly efficient respiratory motion compensated free-breathing coronary MRA using golden-step Cartesian 
acquisition. J Magn Reson Imaging. 2015;41:738-46. https://doi.org/10.1002/ jmri.24602.

40. Bustin $A$, Ginami $G$, Cruz G, et al. Five-minute whole-heart coronary MRA with sub-millimeter isotropic resolution, $100 \%$ respiratory scan efficiency, and 3D-PROST reconstruction. Magn Reson Med. 2019;81:102-15. https:// doi.org/10.1002/mrm.27354.

41. Bustin A, Lima da Cruz G, Jaubert O, Lopez K, Botnar RM, Prieto C. Highdimensionality undersampled patch-based reconstruction (HD-PROST) for accelerated multi-contrast MRI. Magn Reson Med. 2019;81:3705-19. https:// doi.org/10.1002/mrm.27694.

42. Pruessmann KP, Weiger M, Börnert P, Boesiger P. Advances in sensitivity encoding with arbitrary k-space trajectories. Magn Reson Med. 2001;46:63851. https://doi.org/10.1002/mrm.1241

43. Liu J, Peters DC, Drangova M. Method of BO mapping with magnitudebased correction for bipolar two-point Dixon cardiac MRI. Magn Reson Med. 2017;78:1862-9. https://doi.org/10.1002/mrm.26569.

44. Menon RG, Miller GW, Jeudy J, Rajagopalan S, Shin T. Free breathing threedimensional late gadolinium enhancement cardiovascular magnetic resonance using outer volume suppressed projection navigators. Magn Reson Med. 2017;77:1533-43. https://doi.org/10.1002/mrm.26234.

45. Kecskemeti S, Johnson K, François CJ, Schiebler ML, Unal O. Volumetric late gadolinium-enhanced myocardial imaging with retrospective inversion time selection. J Magn Reson Imaging. 2013;38:1276-82. https://doi.org/10.1002/ jmri.24037.

46. Kunze KP, Piccini D, Forman C, et al. 3D Whole-Heart Imaging with Orientation-Independent 2D Image Navigators. In: Society for Magnetic Resonance Angiography, 31st Annual International Conference; 2019. p. 25.

47. Abgral R, Dweck MR, Trivieri MG, et al. Clinical utility of combined FDG-PET/ MR to assess myocardial disease. JACC Cardiovasc Imaging. 2017;10:594-7. https://doi.org/10.1016/j.jcmg.2016.02.029.

48. Hanneman K, Kadoch M, Guo HH, et al. Initial experience with simultaneous 18F-FDG PET/MRI in the evaluation of cardiac sarcoidosis and myocarditis. Clin Nucl Med. 2017;42:e328-34. https://doi.org/10.1097/rlu. 0000000000001669 .

49. Dweck MR, Abgral $\mathrm{R}$, Trivieri MG, et al. Hybrid magnetic resonance imaging and positron emission tomography with Fluorodeoxyglucose to diagnose active cardiac sarcoidosis. JACC Cardiovasc Imaging. 2017;11:94-107. https:// doi.org/10.1016/j.jcmg.2017.02.021.

50. Nensa F, Kloth J, Tezgah E, et al. Feasibility of FDG-PET in myocarditis: comparison to CMR using integrated PET/MRI. J Nucl Cardiol. 2018;25:78594. https://doi.org/10.1007/s12350-016-0616-y.

\section{Publisher's Note}

Springer Nature remains neutral with regard to jurisdictional claims in published maps and institutional affiliations.

Ready to submit your research? Choose BMC and benefit from:

- fast, convenient online submission

- thorough peer review by experienced researchers in your field

- rapid publication on acceptance

- support for research data, including large and complex data types

- gold Open Access which fosters wider collaboration and increased citations

- maximum visibility for your research: over $100 \mathrm{M}$ website views per year

At $\mathrm{BMC}$, research is always in progress.

Learn more biomedcentral.com/submissions 